\title{
Sound propagation and Mach cone in anisotropic hydrodynamics
}

\author{
Martin Kirakosyan $^{1, \mathrm{a}}$, Aleksandr Kovalenko ${ }^{1,2}$, Andrey Leonidov ${ }^{1}$ \\ ${ }^{1}$ P.N. Lebedev Physical Institute, Moscow, Russia \\ ${ }^{2}$ M.V. Lomonosov Moscow State University, Moscow, Russia
}

Received: 26 November 2018 / Accepted: 5 May 2019 / Published online: 22 May 2019

(C) The Author(s) 2019

\begin{abstract}
This letter is based on a kinetic theory approach to anisotropic hydrodynamics. We derive the sound wave equation in anisotropic hydrodynamics and show that a corresponding wave front is ellipsoidal. The phenomenon of Mach cone emission in anisotropic hydrodynamics is studied. It is shown that Mach cone in anisotropic case becomes asymmetric, i.e., in this limit they're two different angles, left and right with respect to the ultrasonic particle direction, which are determined by the direction of ultrasonic particle propagation and the asymmetry coefficient.
\end{abstract}

\section{Introduction}

One of the universal features of highly excited matter created at the early stages of heavy ion collisions is its momentum space anisotropy, extreme at its birth point and, presumably, gradually disappearing in the course of its expansion. Of significant interest are therefore physical phenomena that are directly related to this anisotropy. A natural stylized framework for discussing such phenomena is the socalled anisotropic relativistic hydrodynamics $[1,2]$ in which the momentum anisotropy of evolving "liquid" is built in explicitly. One of the most important phenomena in hydrodynamics is its sound excitation modes and, in particular, the related phenomenon of Mach cone. In this letter we analyze sound propagation and Mach cone emission in anisotropic relativistic hydrodynamics.

The interest to the Mach cone emission in the context of ultra relativistic heavy ion collisions [3-5] was sparked, in particular, by the results on two particle correlations at RHIC $[6,7]$. An alternative explanation of the two-humped structure observed was in terms of Cherenkov radiation of a parton moving with velocity exceeding the speed of gluon propagation in the hot dense medium formed in heavy ion collisions $[8,9]$. However, subsequent studies at LHC did not confirm

a e-mail: markir@cern.ch the existence of the visible two-bump structure in a fragmentation of away side jets $[10,11]$ and, in addition, arguments for significant effects from background suppression at RHIC results were spelled out (see, e.g., a detailed review in [12]). It should also be noted that a two-hump structure may be explained by taking into account geometric fluctuations of initial state in heavy ion collisions $[13,14]$. Despite the fact that at present there is no direct experimental evidence for the Mach cone emission phenomenon, there is still a significant theoretical interest in adjusting a description of this universal phenomenon to the realistic stylized properties of the matter created at the early stages of heavy ion collisions, in particular, its momentum anisotropy. Moreover, there are other observables that could be affected by the Mach cone emission, i.e. the enhancement of low-pt particles away from the quenched jets [15-17].

The analysis in this letter is based on a kinetic theory approach to anisotropic hydrodynamics [2]. In Sect. 2 we derive a sound wave equation in anisotropic hydrodynamics and show that a corresponding wave front becomes ellipsoidal. In Sect. 3 we analyze the phenomenon of Mach cone emission in anisotropic hydrodynamics and show that the cone becomes asymmetric, i. e. there are two different angles, left and right with respect to the ultrasonic particle direction, which are determined by the direction of ultrasonic particle propagation and the asymmetry coefficient.

\section{Sound in anisotropic hydrodynamics}

The phenomenon of sound propagation is analyzed by studying the excitation modes in the linear approximation in fluctuations. Let us perform this analysis for the formulation of anisotropic hydrodynamics based on kinetic theory approach and use the Romatschke-Struckland ansatz [1820] for massless gas:

$$
f(x, p)=f_{i s o}\left(\frac{\sqrt{p^{\mu} \Xi_{\mu \nu} p^{\nu}}}{\Lambda(x)}\right),
$$


where $\left(p^{\mu} \mathbf{\Xi}_{\mu \nu} p^{\nu}=\mathbf{p}^{2}+\xi(x) p_{\|}^{2}\right)$ in the Landau rest frame (LRF) and $\xi$ is an anisotropy parameter which is, generally speaking, a function of the coordinates $x$.

From this simple model one can derive analytical expressions for particle number density $n$ and components of energy-momentum tensor $T^{\mu \nu}$ in the LRF using their standard definitions as the first and second moments of the distribution function [18-20]

$$
\begin{aligned}
T^{00} & =\varepsilon(\Lambda, \xi)=R(\xi) \varepsilon_{i s o}(\Lambda), \\
T^{11} & =T^{22}=P_{\perp}(\Lambda, \xi)=R_{\perp}(\xi) P_{i s o}(\Lambda), \\
T^{33} & =P_{\|}(\Lambda, \xi)=R_{\|}(\xi) P_{i s o}(\Lambda), \\
n(\Lambda, \xi) & =\frac{n_{i s o}(\Lambda)}{\sqrt{1+\xi}},
\end{aligned}
$$

where $\varepsilon_{i s o}(\Lambda), P_{i s o}(\Lambda)$ and $n_{i s o}(\Lambda)$ are isotropic energy density, pressure and particle number density respectively obtained from isotropic distribution function $f_{i s o}$ and the dependence on the anisotropy parameter is factored out for all the variables considered. The dependence of the factors $R, \perp, \|$ on the anisotropy parameter $\xi$ is given by [20,21]:

$$
\begin{aligned}
R(\xi) & =\frac{1}{2}\left(\frac{1}{1+\xi}+\frac{\arctan \sqrt{\xi}}{\sqrt{\xi}}\right), \\
R_{\perp}(\xi) & =\frac{3}{2 \xi}\left(\frac{1+\left(\xi^{2}-1\right) R(\xi)}{1+\xi}\right), \\
R_{\|}(\xi) & =\frac{3}{\xi}\left(\frac{(\xi+1) R(\xi)-1}{1+\xi}\right) .
\end{aligned}
$$

In what follows it turns out convenient to introduce the following parametrization for the quantities involved in describing the properties of an anisotropic fluid in terms of a fourvector $u_{\mu}(x)$ and a rapidity $\vartheta(x)$ :

$U^{\mu}=\left(u_{0} \cosh \vartheta, u_{x}, u_{y}, u_{0} \sinh \vartheta\right)$,

$X^{\mu}=\left(u_{\perp} \cosh \vartheta, \frac{u_{0} u_{x}}{u_{\perp}}, \frac{u_{0} u_{y}}{u_{\perp}}, u_{\perp} \sinh \vartheta\right)$,

$Y^{\mu}=\left(0,-\frac{u_{y}}{u_{\perp}}, \frac{u_{x}}{u_{\perp}}, 0\right)$,

$Z^{\mu}=(\sinh \vartheta, 0,0, \cosh \vartheta)$,

where $U^{\mu}$ is the 4-velocity vector that describes the hydrodynamic flow, $Z^{\mu}$ defines the direction of the longitudinal axis and $X^{\mu}$ and $Y^{\mu}$ define axes in the transverse plane and the four-vector $u_{\mu}$ satisfies $u_{\mu}(x) u_{0}^{2}=1+u_{x}^{2}+u_{y}^{2}$ (4vectors $U$ are normalized so that $U_{\mu} U^{\mu}=1$, the same holds for $X, Y$ and $Z$ ), $\vartheta$ is the fluid rapidity. In the LRF one has $U^{\mu}=(1,0,0,0), Z^{\mu}=(0,0,0,1)$.
Assuming conservation of energy-momentum tensor $T^{\mu \nu}$ and particle current $j^{\mu}$ one gets:

$$
\begin{aligned}
\partial_{\mu} T^{\mu \nu} & = & 0 \\
\partial_{\mu} j^{\mu} & = & 0 .
\end{aligned}
$$

where

$$
\begin{aligned}
T^{\mu \nu} & =\left(\varepsilon+P_{\perp}\right) U^{\mu} U^{\nu}-P_{\perp} g^{\mu \nu}-\left(P_{\perp}-P_{\|}\right) Z^{\mu} Z^{\nu}, \\
j^{\mu} & =n U^{\mu} .
\end{aligned}
$$

Linearization of $(13,14)$ leads to equations describing propagation of sound [22]. To derive sound equation for the case under consideration let us expand the 4-velocities $U$, $Z$, particle number density, energy and momentum densities with respect to a temperature parameter $\Lambda(x)=\Lambda^{(0)}+$ $\Lambda^{(1)}(x)$ to the leading order in gradients:

$$
\begin{aligned}
U^{\mu} & =U^{(0) \mu}+U^{(1) \mu}(x), \\
Z^{\mu} & =Z^{(0) \mu}+Z^{(1) \mu}(x), \\
\varepsilon & =\varepsilon^{(0)}+\varepsilon^{(1)}(x), \\
P_{\perp, \|} & =P_{\perp, \|}^{(0)}+P_{\perp, \|}^{(1)}(x), \\
n & =n^{(0)}+n^{(1)}(x) .
\end{aligned}
$$

Let us now move to the frame in which $U^{(0)}=(1,0,0,0)$ and $Z^{(0)}=(0,0,0,1)$. That gives us, through $U^{\mu} Z_{\mu}=0$, same relations between components of $U^{(1)}$ and $Z^{(1)}$. Let us also introduce the following notations:

$$
\begin{aligned}
\varepsilon^{(1)}(x) & =c_{\varepsilon} n^{(1)}(x), \\
P_{\perp}^{(1)}(x) & =c_{\perp} n^{(1)}(x), \\
P_{\|}^{(1)} & =c_{\|} n^{(1)}(x) .
\end{aligned}
$$

Assuming smallness of the gradients of the anisotropy parameter $\xi$ and limiting our consideration to transverse fluctuations with respect to $u_{\mu}^{(0)}$ we obtain, using (1), (13) and (14), the following sound equation

$\left(c_{\perp} \partial_{x}^{2}+c_{\perp} \partial_{y}^{2}+c_{\|} \partial_{z}^{2}-c_{\varepsilon} \partial_{t}^{2}\right) n^{(1)}=0$.

From (25) one can see that it leads to an ellipsoidal front of a sound wave. Coefficients $c_{\|, \perp \ldots}$ may be obtained obtain from representation of (22)-(24) as a series of anisotropydependent density parameter (taking $\Lambda=\Lambda^{0}+\Lambda^{(1)}$ ), using (2) and keeping terms of order of $\Lambda^{(1)}$.

$$
\begin{aligned}
c_{\varepsilon} & =\sqrt{1+\xi} R(\xi) A\left(\Lambda_{0}\right) \\
c_{\perp} & =\frac{1}{3} \sqrt{1+\xi} R_{\perp}(\xi) A\left(\Lambda_{0}\right),
\end{aligned}
$$




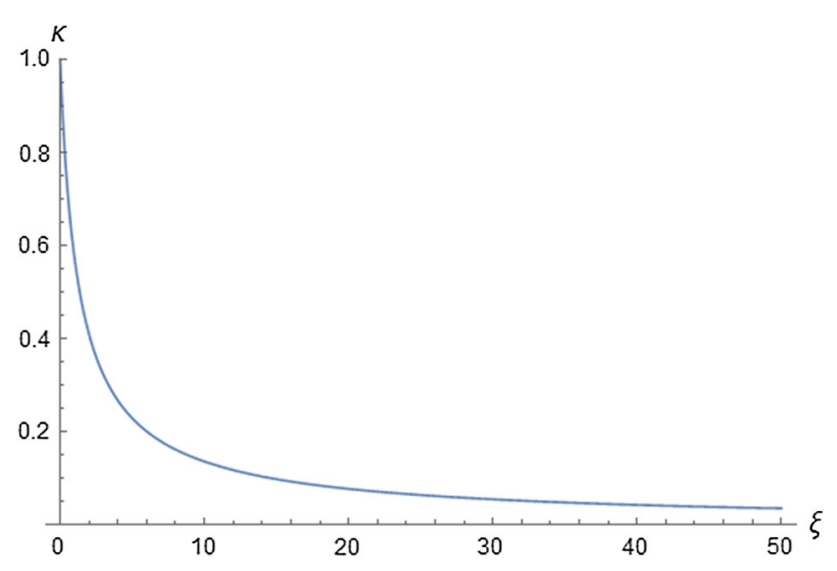

Fig. $1 \kappa(\xi)$

$c_{\|}=\frac{1}{3} \sqrt{1+\xi} R_{\|}(\xi) A\left(\Lambda_{0}\right)$

where $A\left(\Lambda_{0}\right)=\frac{\partial \varepsilon_{i s o}\left(\Lambda_{0}\right) / \partial \Lambda}{\partial n_{\text {iso }}\left(\Lambda_{0}\right) / \partial \Lambda}$

Introducing a notation $\kappa=c_{\|} / c_{\perp}$ and using a relation $c_{\varepsilon}=2 c_{\perp}+c_{\|}$valid in the ultra-relativistic case, we can rewrite $(25)$ in the following form:

$\left(\partial_{x}^{2}+\partial_{y}^{2}+\kappa \partial_{z}^{2}\right) n^{(1)}=(\kappa+2) \partial_{t}^{2} n^{(1)}$.

In the isotropic limit $\kappa=1$ equation (27) reduces to the standard isotropic ultrarelativistic sound equation. Let us note that $\kappa$ may be written as $\kappa=\frac{P_{\|}}{P_{\perp}}$ and, therefore, $\kappa$ is the anisotropy-related quantity that is, in principle, observable. A dependence of $\kappa$ on the anisotropy parameter is shown in Fig. 1.

\section{Mach cone}

One of the characteristic phenomena in hydrodynamics is the appearance of the Mach cone, an expanding shock wave that is generated by an ultrasonic body propagating in a medium. Its properties depend on the ratio of the local flow velocity $v$ and the speed of sound in the medium $c_{s}$ - the Mach Number $(\mathrm{MN})$. The Mach cone appears for $M N>1$. For isotropic theories there is a well known formula for Mach angle, an angle with respect to the direction of propagation of ultrasonic particle at which the shock wave is emitted: $\sin \theta_{M 0}=c_{S} / v$, in ultrarelativistic case $\theta_{M 0}=\arcsin 1 / 3$. Obviously, in anisotropic theory there is no such simple relation. First, the base of the cone is no longer a circle, but an ellipse, i.e. the shape of the sound wave front is ellipsoidal (see Eq. 25). Second, the front is symmetric in a plane transversal to direction of anisotropy, in the wave equation (27) it is Oxy plane. Thus, one may consider 2D-problem instead of 3D-problem and fix any axis in the transverse plane

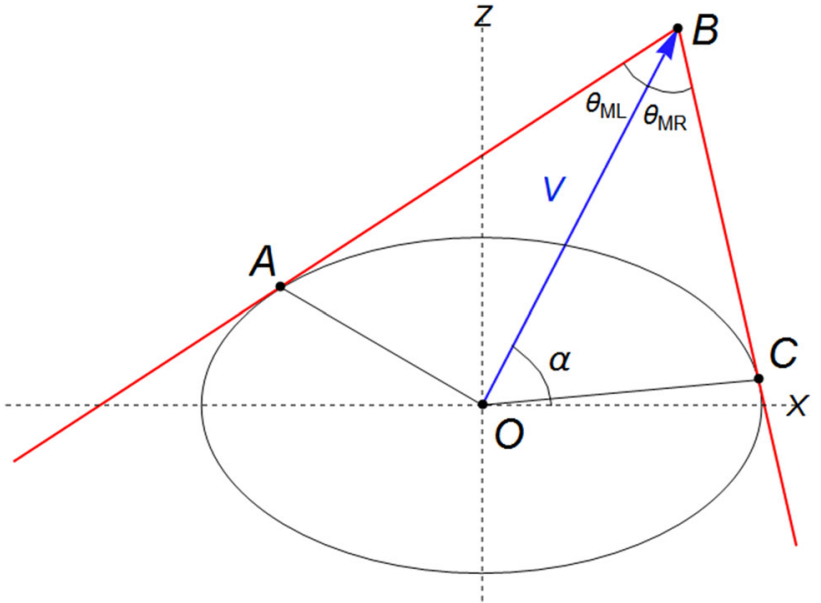

Fig. 2 Representation of the Mach cone

(say $O x$ ). In Fig. 2 we plot a 2-D slice of a full 3-D picture and show a particle moving with velocity $v$ from $O$ in the plane $O x z$ at an angle $\alpha$ to the $O x$ axis. With $v / c_{s}>1$ there appears a Mach cone, which is formed by tangents to the ellipsoid. In Fig. 2 the particle is at point $B$ and $A B, C B$ are tangents to the ellipsoid, $\theta_{M R}, \theta_{M L}$ are the Mach angles.

Having an anisotropic sound with $c_{z}=\sqrt{\kappa} / \sqrt{\kappa+2}, c_{x}=$ $1 / \sqrt{\kappa+2}$ we get the following formula for the ellipse (say, upper part $z>0$ ) and tangent $A B$ as functions of $x$ :

$f_{1}(x)=c_{z} \sqrt{1-\frac{x^{2}}{c_{x}^{2}}}$
$f_{2}(x)=v \sin \alpha+(x-v \cos \alpha) \tan \left(\alpha-\theta_{M}\right)$.

and thus obtain a quadratic equation for $x$, so that description of the Mach cone is fixed as the condition of existence of its roots. We get:

$\theta_{M L}=\arctan \left[\frac{\sin \alpha \cos \alpha(\kappa-1)+\sqrt{\left(\kappa \cos ^{2} \alpha+\sin ^{2} \alpha\right)(\kappa+2)-\kappa}}{(\kappa+2)-\kappa \sin ^{2} \alpha-\cos ^{2} \alpha}\right]$,

$\theta_{M R}=\arctan \left[\frac{\sin \alpha \cos \alpha(1-\kappa)+\sqrt{\left(\kappa \cos ^{2} \alpha+\sin ^{2} \alpha\right)(\kappa+2)-\kappa}}{(\kappa+2)-\kappa \sin ^{2} \alpha-\cos ^{2} \alpha}\right]$.

Here we define two different Mach angles $\theta_{M L}, \theta_{M R}$ which characterize the whole Mach cone (not only its 2Dslice). It should be noted that if $\kappa=1$ (no anisotropy) then $\theta_{M L}=\theta_{M R}=\arctan 1 / \sqrt{2}$, the standard expression for the Mach angle in the relativistic isotropic hydrodynamics (Figs. $3,4)$. 
Fig. 3 Mach angles $\theta_{M L}$ and $\theta_{M R}$ as functions of $\alpha, \theta_{M 0}$ is a Mach angle in isotropic case. a $\kappa=0.01, \mathbf{b} \kappa=0.1, \mathbf{c} \kappa=0.5$, d $\kappa=0.9$ $\theta_{M R}$ as functions of $\kappa, \theta_{M 0}$ is a Mach angle in isotropic case. a $\alpha=\pi / 12, \mathbf{b} \alpha=\arcsin 1 / 3, \mathbf{c}$ $\alpha=\pi / 6, \mathbf{d} \alpha=\pi / 3$
Fig. 4 Mach angles $\theta_{M L}$ and
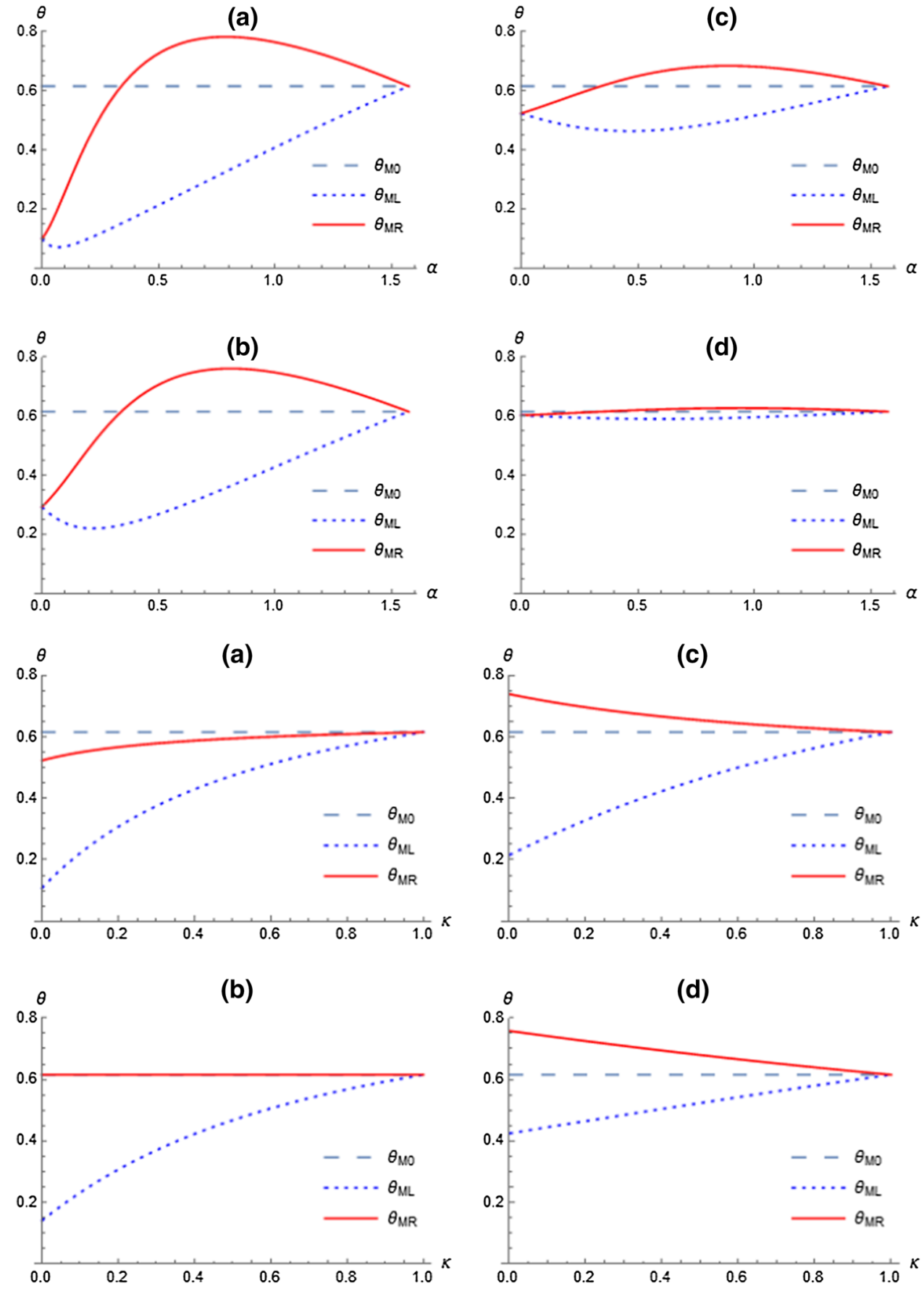

and it's relation to observables in ultrarelativistic heavy ion collisions (angular flow asymmetry, for instance).

In this letter we have developed an analytical description of the Mach cone in relativistic anisotropic hydrodynamics. We derived sound wave equation in anisotropic hydrodynamics and demonstrated that in anisotropic case a wave front turns out to be ellipsoidal. For Mach cone emission as it was shown there are two different angles, left and right with respect to ultrasonic particle direction.

In future we plan to develop a model for isotropization using the combination of analytical and numerical methods
Acknowledgements Authors of this paper, Andrey Leonidov and Aleksandr Kovalenko were supported by the RFBR Grant 18-02-40069mega.

Data Availability Statement This manuscript has no associated data or the data will not be deposited. [Authors' comment: This is a theoretical article. So there is no data to provide.]

Open Access This article is distributed under the terms of the Creative Commons Attribution 4.0 International License (http://creativecomm 
ons.org/licenses/by/4.0/), which permits unrestricted use, distribution, and reproduction in any medium, provided you give appropriate credit to the original author(s) and the source, provide a link to the Creative Commons license, and indicate if changes were made. Funded by SCOAP ${ }^{3}$.

\section{References}

1. G.S. Denicol et al., Shear-bulk coupling in nonconformal hydrodynamics. Phys. Rev. C 90, 04490 (2014). https://doi.org/10.1103/ PhysRevC.90.044905

2. M. Martinez, M. Strickland, Non-boost-invariant anisotropic dynamics. Nucl. Phys. A 856, 68-87 (2011). https://doi.org/10. 1016/j.nuclphysa.2011.02.003

3. J. Casalderrey-Solana, E.V. Shuryak, D. Teaney, Conical flow induced by quenched qcd jets. Nucl. Phys. A. 774, 577-580 (2006). https://doi.org/10.1088/1742-6596/27/1/003

4. Victor Roy, A.K. Chaudhuri, Equation of state dependence of mach cone like structures in au+au collisions. J. Phys. G. 37, 035105 (2010). https://doi.org/10.1088/0954-3899/37/3/035105

5. T. Renk, J. Ruppert, Three-particle azimuthal correlations and mach shocks. Phys. Rev. C 76, 014908 (2007). https://doi.org/10. 1103/PhysRevC.76.014908

6. M. M. Aggarwal et al. (STAR collaboration). Azimuthal di-hadron correlations in $\mathrm{d}+\mathrm{au}$ and au+au collisions at $\sqrt{s_{N N}}=200 \mathrm{gev}$ from star. Phys. Rev. C. 82, 024912 (2010). https://doi.org/10.1103/ PhysRevC.82.024912

7. A. Adare et al. (PHENIX collaboration). Transition in yield and azimuthal shape modification in dihadron correlations in relativistic heavy ion collisions. Phys. Rev. Lett. 104, 252301 (2010). https:// doi.org/10.1103/PhysRevC.82.024912

8. I.M. Dremin, In-medium qcd and cherenkov gluons. Eur. Phys. J. C. 56, 81-86 (2008). https://doi.org/10.1140/epjc/ s10052-008-0627-1

9. I.M. Dremin et al., Cherenkov glue in opaque nuclear medium. Nucl. Phys. A 826, 190-197 (2009). https://doi.org/10.1140/epjc/ s10052-008-0627-1

10. G. Aad et al. (ATLAS collaboration).Measurement of inclusive jet charged-particle fragmentation functions in $\mathrm{pb}+\mathrm{pb}$ collisions at $\sqrt{s_{N N}}=2.76$ tev with the atlas detector. Phys. Lett. B 739, 320 (2014). https://doi.org/10.1016/j.physletb.2014.10.065
11. S. Chatrchyan et al. (CMS collaboration). Measurement of jet fragmentation in pbpb and pp collisions at $\sqrt{s_{N N}}=2.76$ tev. Phys. Rev.C 90, 024908 (2014). https://doi.org/10.1103/PhysRevC.90.024908

12. C. Nattrass et al., Disappearance of the mach cone in heavy ion collisions. Phys. Rev. C. 94, 011901(R) (2016). https://doi.org/10. 1103/PhysRevC.94.011901

13. B. Schenke, S. Jeon, C. Gale, Elliptic and triangular flow in eventby-event $\mathrm{D}=3+1$ viscous hydrodynamics. Phys. Rev. Lett. 106, 042301 (2011). https://doi.org/10.1103/PhysRevLett.106.042301

14. G.-L. Ma, X.-N. Wang, Jets, mach cones, hot spots, ridges, harmonic flow, dihadron, and $\gamma$-hadron correlations in high-energy heavy-ion collisions. Phys. Rev. Lett. 106, 162301 (2011). https:// doi.org/10.1103/PhysRevLett.106.162301

15. S. Chatrchyan et al. (CMS Collaboration). Observation and studies of jet quenching in pbpb collisions at nucleon-nucleon center-ofmass energy $=2.76$ tev. Phys. Rev. C, 84024906 (2011). https:// doi.org/10.1103/PhysRevC.84.024906

16. S. Chatrchyan et al. (CMS Collaboration). Measurement of jet fragmentation into charged particles in $\mathrm{pp}$ and $\mathrm{pbpb}$ collisions at $\sqrt{s_{N N}}=2.76$ tev. JHEP 10 p. 087 (2012). https://doi.org/10.1007/ JHEP10(2012)087

17. Y. Tachibana, T. Hirano, Momentum transport away from a jet in an expanding nuclear medium. Phys. Rev. C 90, 021902 (2014). https://doi.org/10.1103/PhysRevC.90.021902

18. P. Romatschke, M. Strickland, Collective modes of an anisotropic quark-gluon plasma. Phys. Rev. D 68, 036004 (2003). https://doi. org/10.1103/PhysRevD.68.036004

19. P. Romatschke, M. Strickland, Collective modes of an anisotropic quark-gluon plasma ii. Phys. Rev. D 70, 116006 (2004). https:// doi.org/10.1103/PhysRevD.70.116006

20. M. Strickland, Anisotropic hydrodynamics: three lectures. Acta Phys. Polon. B 45, 2355 (2014). https://doi.org/10.5506/ APhysPolB.45.2355

21. W. Florkowski, R. Ryblewski, Highly-anisotropic and stronglydissipative hydrodynamics for early stages of relativistic heavyion collisions. Phys. Rev. C 83, 034907 (2011). https://doi.org/10. 1103/PhysRevC.83.034907

22. L.D. Landau, E.M. Lifshitz, Fluid Mechanics, vol. 6 (Elsevier Science, Oxford, 1987) 